\title{
Producción de leche en vacas mestizo Holstein (Bos taurus L.,) pastoreadas en paraderas nativas en comparación con las suplementadas con borra de cerveza y maíz amarillo

\author{
Milk production in Holstein mestizo cows (Bos taurus L.) grazed on native stands compared to those \\ supplemented with beer gills and yellow corn
}

Blanco-Callancho Florentino $^{1 *}$, Loza-Murguia Manuel Gregorio ${ }^{2,3}$, Achu-Nina Cristóbal ${ }^{1}$, Chura- Limachi Felipe ${ }^{1}$

\begin{tabular}{l} 
Datos del Artículo \\
\hline \\
1 Universidad Católica Boliviana San Pablo- \\
UCBSP. Unidad Académica Campesina \\
Tiahuanacu UAC-T. \\
Ingeniería Zootécnica. Km 74. Carretera \\
Internacional La Paz-Desaguadero. \\
Tel +591-2-2895100. La Paz, Estado \\
Plurinacional de Bolivia. \\
2 Universidad Católica Boliviana San Pablo- \\
UCBSP, Unidad Académica Campesina \\
Carmen Pampa-UAC-CP, Ingeniería \\
Agronómica. Coroico-Nor Yungas-La Paz, \\
Estado Plurinacional de Bolivia. Tel \\
+591(2)8781991. \\
3 Departamento de Enseñanza e Investi \\
gación en Bioquímica \& Microbiología- \\
DEI\&BM. Unidad Académica Campesina \\
Carmen Pampa-UAC-CP. \\
*Dirección de contacto: \\
Universidad Católica Boliviana San Pablo - \\
UCBSP. Unidad Académica Campesina \\
Tiahuanacu UAC-T. \\
Ingeniería Zootécnica. Km 74. Carretera \\
Internacional La Paz-Desaguadero. \\
Tel: +591-73275606. \\
Florentino Blanco-Callancho \\
E-mail address : blanco.1989.z@gmail.com \\
\hline \\
\hline
\end{tabular}

\section{Palabras clave: \\ Vacas mestizo Holstein, \\ suplemento, \\ pradera nativa, \\ producción de leche, \\ raciones.}

\section{Resumen}

La presente investigación se realizó en un sector de la cuenca lechera, Cantón Patamanta del Municipio de Pucarani, con el objetivo de determinar la producción de leche en vacas mestizas Holstein (VMH) pastoreadas en pradera nativa (PPN), y se comparó con las suplementadas con borra de cerveza (BC) y maíz amarillo (MA). 9 VMH de peso vivo (PV) de 375 $\mathrm{kg}$, de 5 a 6 años de edad, 3 a 5 meses post parto, de segundo periodo de lactancia, clínicamente sanas y libres de parásitos. La evaluación se realizó entre los meses de abril, mayo y junio, los animales estaban estacados con una soga de $3.4 \mathrm{~m}$, se los recorría dos veces al día, por la noche se los estabulaba. Las raciones para los tres grupos de 3 VMH fueron: i) PPN sin suplemento ii) PPN + 6.0 kg de BC iii) PPN + 1.59 kg de BC + 5.46 kg MA, el suplemento se preparó en recipientes que contenía $0.2 \mathrm{~kg}$ de sal y agua corriente $15 \mathrm{~L}$, el suplemento era disuelto en agua y sal, el grupo (i), era agua y sal en su bebedero, la suplementación se les proporcionaba dos veces al día, por la mañana y al finalizar el día, el diseño aplicado fue completamente el azar, para la comparación de medias se utilizó DUNCAN y ANOVA. Se evaluó, la producción de leche y parámetros de calidad de la leche cruda, el contenido de proteína (CP), contenido de grasa (CG), solidos totales (ST), además el consumo de agua requerida para la producción de $1 \mathrm{~kg}$ de leche cruda, el costo de producción de leche.

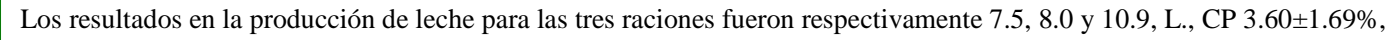
$4.32 \pm 1.80 \%$, y $4.06 \pm 0.93 \%$, el CG $2.91 \pm 0.27 \%$, $3.50 \pm 0.79 \%$, y $3.81 \pm 0.69 \%$, ST $12.69 \pm 0.63 \%$, $13.50 \pm 0.73 \%$, y $11.34 \pm 0.49 \%$, la cantidad de agua requerida para la producción de $1 \mathrm{~kg}$ de leche $13,16.2$ y $12 \mathrm{~L}$, los costos de producción 1.70, 1.73, 2.10 Bs. La producción de leche, con la ración III fue mayor en relacion a las otras dos, lo que nos indica que esta ración brinda aporte energético y proteico necesario en la suplementación, cubriendo sus requerimientos diarios. En cuanto a la calidad de la leche, se observa, CP, CG, y ST, mejoro notablemente con la ración III, lo que se asocia al consumo de agua, que también es menor en esta ración, en comparación las otras dos, los costos en la ración III, se incrementa 0.4 Bs. Con estos datos la producción de leche no afecta su calidad, si bien el costo se incrementa, que pese a las condiciones de las familias que se dedican a esta actividad pecuaria serian beneficiarias con su incremento, lo que contribuiría a sus ingresos económicos, además mejorarían el balance energético de las $\mathrm{VMH}$, que a la larga, sería un aporte significativo en la producción de esta materia prima que al ser un alimento básico, se requiere que su producción en cantidad y calidad sea superior a medida que se mejore la alimentación de los animales de esta área rural.

(C) 2018. Journal of the Selva Andina Animal Science. Bolivia. Todos los derechos reservados.

\section{Abstract}

The present investigation was carried out in a sector of the milk basin, Patamanta Canton of the Municipality of Pucarani, with the objective of determining milk production in Holstein crossbred cows (HCC) grazed in native pasture (GNP), and compared with those supplemented with beer blot (BB) and yellow corn (YC). $9 \mathrm{HCC}$ of live weight (LW) of $375 \mathrm{~kg}$, from 5 to 6 years of age, 3 to 5 months postpartum, of second lactation period, clinically healthy and free of parasites. The evaluation was carried out between the months of April, May and June, the animals were staked with a rope of $3.4 \mathrm{~m}$, they were traversed twice a day, at night they were stabled. The rations for the three groups of 3 HCC were: i) GNV without
Recibido abril, 2017.

Devuelto diciembre 2017

tado julio, 2018.

Disponible en línea, octubre, 2018. 


\begin{tabular}{c}
\hline Editado por: \\
Selva Andina \\
Research Society \\
\hline
\end{tabular}

supplement ii) $\mathrm{HCC}+6.0 \mathrm{~kg}$ of $\mathrm{BB}$ iii) $\mathrm{GNP}+1.59 \mathrm{~kg}$ of $\mathrm{BB}+5.46 \mathrm{~kg} \mathrm{YC}$, the supplement was prepared in containers containing $0.2 \mathrm{~kg}$ of salt and running water $15 \mathrm{~L}$, the supplement was dissolved in water and salt, the group (i), was water and salt in its drinking fountain, the supplementation was provided twice a day, in the morning and at the end of the day, the applied design It was completely random, for the comparison of means, DUNCAN and ANOVA were used. Milk production and quality parameters of raw milk, protein content (PC), fat content (FC), total solids (TS), as well as the water consumption required for the production of $1 \mathrm{~kg}$ of milk were evaluated. Raw milk, the cost of milk production. The results in milk production for the three rations were respectively 7.5, 8.0 and 10.9, L., PC $3.60 \pm 1.69 \%, 4.32 \pm 1.80 \%$, and $4.06 \pm 0.93 \%$, FC $2.91 \pm 0.27 \%, 3.50 \pm 0.79 \%$, and $3.81 \pm 0.69 \%$, TS $12.69 \pm 0.63 \%, 13.50 \pm 0.73 \%$, and $11.34 \pm$ $0.49 \%$, the amount of water required for the production of $1 \mathrm{~kg}$ of milk 13, 16.2 and $12 \mathrm{~L}$, production costs $1.70,1.73$, $2.10 \mathrm{Bs}$. Milk production, with ration III was higher in relation to the other two, which indicates that this ration provides energy and protein intake necessary in the supplementation, covering their daily requirements. Regarding the quality of the milk, PC, FC, and TS, improved notably with ration III, which is associated with water consumption, which is also lower in this ration, compared to the other two, costs in ration III, increases 0.4 Bs. With these data milk production does not affect its quality, although the cost increases, that despite the conditions of the families that are dedicated to this livestock activity would be beneficiaries with its increase, which would contribute to their economic income, would also improve the energy balance of the HCC, which in the long run, would be a significant contribution in the production of this raw material which, being a staple food, requires that its production in quantity and quality be superior as the feeding of animals in this rural area improves.

(C) 2018. Journal of the Selva Andina Animal Science. Bolivia. All rights reserved.

\section{Introducción}

La crianza del ganado bovino lechero en Bolivia, se constituye en una de las actividades de mayor importancia económica para los grandes, medianos y pequeños productores dedicados al rubro, así, en los últimos años esta actividad fue adquiriendo mayor importancia por los ingresos económicos que genera para las familias.

Estudios realizados por la Organización de las Naciones Unidas para la Alimentación y la Agricultura (FAO) acerca de la situación de la lechería en América Latina y el Caribe en 2011, reporto que, de los 19 países América Latina, Bolivia está en el décimo octavo lugar con aproximadamente 11000 productores, lo que significa que está en el décimo sexto lugar de productores de los países evaluados ${ }^{1}$

Es necesario señalar que en el departamento de $\mathrm{La}$ Paz la producción promedio de leche es baja, y en base a experiencias locales, han logrado mejorar los rendimientos a $6.5 \mathrm{~L} / \mathrm{vaca} / \mathrm{dí}$, con mejoras en alimentación, infraestructuras y manejo de pastizales. ${ }^{2}$
La producción ganadera en la región altiplánica de La Paz está incrementándose notablemente en los últimos años, convirtiéndose en zona principal de abastecimiento de leche para la ciudad de $\mathrm{La} \mathrm{Paz}^{3}$. El "Cordón Lechero" en La Paz, comprende parte de las provincias del Altiplano Norte y Sur, abarcando 5 provincias: Omasuyos, Los Andes, Ingavi, Murillo y Aroma, que comprenden 13 secciones municipales, con aproximadamente 131 comunidades que se dedican a la ganadería lechera. ${ }^{4}$

La producción lechera en sistemas especializados se relaciona con la capacidad genética de los animales, su manejo, estado sanitario del hato, plan de alimentación, considerando este último factor, como la cantidad de nutrientes consumidos, como principal limitante para una producción eficiente. ${ }^{5,6}$ El consumo de materia seca (CMS) en nutrición bovina es vital, especialmente en animales en pastoreo, pues permite determinar la cantidad de nutrientes disponibles para su salud, reproducción y producción ${ }^{7}$, la estimación del CMS, además de ser indicador de capacidad productiva y estado nutri- 
cional en pastoreo, es necesario en estos sistemas de producción para evitar subalimentación, lo que restringiría la producción e incrementaría los costos, debido a los bajos índices de transformación del alimento en productos de origen animal. ${ }^{8,6}$

Lo anterior puede ser mejorado con suplementación de forrajes picados, como maíz, suministrados en comederos, mezclados como ración total con balanceados que incrementan la energía para la lactación, mejorando el consumo total de los alimentos, que se observó en vacas lecheras en Australia tropical. ${ }^{9,10}$ La borra de cerveza (BC) también conocida como afrecho o cebadilla, subproducto de la industria cervecera, resultante del proceso de prensado y filtración del mosto, obtenido tras la sacarificación del grano de cereal (cebada, básicamente) malteado. Un producto húmedo cuyo contenido en materia seca (MS) 20 - 25\% $\%^{11}$, con una energía metabolizable (EM) $2.9 \mathrm{Mcal} / \mathrm{kg}$. Con degradabilidad efectiva de proteína baja $(50 \%)$, siendo su velocidad de $7 \% / \mathrm{h}$, un alimento de elevado contenido proteico, proteína que escapa, en buena parte de la degradación ruminal, utilizada en alimentación de rumiantes, especialmente vacas lecheras. Por su alto contenido de fibra, su utilización en monogástricos es limitada, sin embargo es útil en cerdos y aves. ${ }^{12}$ La borra húmeda de cervecería (BHC), otro subproducto de la industria cervecera, por su alta concentración proteica y fibra, puede ser muy beneficiosa en la dieta de rumiantes, apoyando la función del rumen y la producción de leche y carne. El contenido de proteína cruda (PC), oscila entre 25 a $34 \%$, localizada principalmente en el germen del grano ya utilizado, se digiere de manera limitada en el rumen y en mayor cantidad en el intestino delgado.

Maíz amarillo (MA), subproducto de la industrialización de productos de origen vegetal destinados exclusivamente a la alimentación animal. Posee 88 67
\% MS, $3.3 \mathrm{Mcal} / \mathrm{kg} 8.8$ de PC, 2.6 de fibra cru$\mathrm{da}^{13,12}$, señalan que es uno de los principales ingredientes para el ganado bovino, por su alto valor energético, palatabilidad, escasa variabilidad de su composición química y bajo contenido en factores antinutritivos, carece lisina y triptófano. La fracción nitrogenada del grano tiene una baja proporción de proteínas metabólicas solubles (albuminas y globulina $6 \%$ ), y altas de proteínas de reservas (40\% de glutelina, $54 \%$ prolamina), esta última muy insoluble, responsable relativamente baja de la degradabilidad de proteína en rumiantes $45 \%$.

En relacion a lo mencionado y en virtud a la falta de información el objetivo fue evaluar la producción de la leche en vacas mestizo Holstein (Bos taurus L.,) pastoreadas en pradera nativa en comparación con las suplementadas con borra de cerveza y maíz amarrillo.

\section{Materiales y métodos}

La investigación se realizó en el cantón Patamanta, primera Sección Municipal de Pucarani, Provincia Los Andes del departamento de La Paz. Geográficamente se encuentra ubicada entre los paralelos $16^{\circ} 32^{\prime}$ a $16^{\circ} 05^{\prime}$ de Latitud Sur y $68^{\circ} 43^{\prime}$ a $68^{\circ} 09^{\prime}$ de Longitud Oeste, a una altura de 3838 metros sobre el nivel del mar. ${ }^{14}$ La cuenca lechera de la provincia Los Andes, geográficamente se encuentra ubicada en el Altiplano Norte, comprendido entre los municipios de Pucarani, Laja, Batallas y Puerto Pérez, con una temperatura promedio de $7.5^{\circ} \mathrm{C}$, precipitación pluvial promedio es de $576 \mathrm{~mm} / \mathrm{año}$, registra en los meses diciembre, enero, febrero y marzo. $^{14}$

La pradera nativa utilizada para el pastoreo "chillihuar-gramadal", presento la composición florística de Festuca dolichophylla, Nassella sp., Bromus 
cathárticus, Deyuxia sp., Hordeum muticum, Alchemilla pinnata, Poa annua, Muhlenbergia fastigiata.

En coordinación con la directiva de la Asociación de Lecheros Vacunos de Patamanta (ALVAPA), se identificó tres hatos de ganado bovino lechero, se las denominó unidades familiares de producción (UFP's). Los criterios de selección fueron: i) animales pastoreados en la pradera nativa (amarre en estaca), ii) animales pastoreados en la pradera nativa y suplementadas con 6.0 a $8.0 \mathrm{~kg}$ de BC por animal, iii) animales estabulados durante la noche, iv) dos ordeños de la leche/día, v) ordeño, manual y con estimulo de ternero.

Se seleccionaron 9 VMH multíparas, en la segunda etapa de lactancia con peso vivo (PV) $375 \mathrm{~kg}$, tres a cuatro partos, la identificación se realizó con caravanas plásticas previamente enumeradas, con fin de registrar los animales para su seguimiento en producción de leche. La selección de VMH se consideró, i) lactancia de 3 meses post parto, ii) número de parto entre 3 a 4, iii) grado de condición corporal de 2 a 3 clínicamente sanos y libres de parásitos.
La investigación se realizó durante los meses de abril, mayo y junio que corresponde al periodo de transición época de lluvia a época seca del año 2015, época en que alimentan sus vacas lecheras en praderas nativas reducidas por motivos de sembradíos de forrajes y otros cultivos agrícolas.

La desparasitación interna y externa, se realizó con Paramec Gold LA+ADE a una dosis de $1.0 \mathrm{~mL} / 50$ kg PV.

El pesaje se realizó, primero a los 7 días previa al inicio de los tratamientos con el propósito de obtener dato del peso promedio de VMH para realizar la formulación de raciones, el segundo pesaje a la conclusión de los tratamientos, para cuantificar el comportamiento de incremento o descenso de PV, ambos en ayunas en las mañanas (7:00 a.m.) en una balanza digital (con capacidad de $1500 \mathrm{~kg}$ ).

Consumo de Materia Seca (MS) durante el pastoreo. A través de la diferencia de cantidad disponible del pastizal previa y después del consumo de la pradera nativa por el sistema de estacado con soga de VMH. ${ }^{15}$

Tabla 1 Distribución de unidades experimentales

\begin{tabular}{lcc}
\hline \multicolumn{1}{c}{ Tratamientos } & Nr repeticiones & Días de tratamiento \\
\hline $\begin{array}{l}\text { Ración I: Pradera nativa (sin suplemento) } \\
\begin{array}{l}\text { Ración II: Pradera nativa suplementado con borra de cerveza } \\
\text { (testigo) }\end{array}\end{array}$ & 3 & 80 \\
$\begin{array}{l}\text { Ración III: Pradera nativa suplementados con borra de cerveza y } \\
\text { maíz amarillo (balanceado) }\end{array}$ & 3 & 80 \\
\hline
\end{tabular}

Periodo de acostumbramiento. Durante 10 días previos se ofreció la ración formulada a las $\mathrm{VMH}$, incrementándose gradualmente el alimento a cada grupo, con el fin de evitar dificultades en la degradación de diversos nutrientes asimilados.

Alimentación de $V M H$. La ración estaba formulada de la siguiente forma: i) Ración I (sin suplemento), este grupo no se realizó ningún tipo de preparado, solo fue PPN. ii) Ración II (testigo), $6 \mathrm{~kg} \mathrm{BC,} 2 \mathrm{~kg}$ de sal en 15 L de agua potable. iii) Ración III (balanceado), $1.58 \mathrm{~kg}$ de BC y $5.48 \mathrm{~kg}$ de MA picado, $0.2 \mathrm{~kg}$ sal, en $15 \mathrm{~L}$ agua potable, la mezcla se hizo en recipientes de polietileno de $20 \mathrm{~L}$ para las raciones II y III.

Las 9 VMH fueron PPN, durante 8 h/día, estacados con una cuerda de $3.4 \mathrm{~m}$ y la rotación se la realizaba 
2 veces al día. Mientras la ración formulada para los tratamientos II y III, se administró en dos horarios del día por la mañana de 7:30 a 8:00 a.m. y por la tarde de 17:30 a 18:00 p.m., se preparaba el momento de la alimentación en los comederos. Por la noche se estabulaban las VMH, realizándose los cuidados sanitarios revisando la presencia de heridas o enfermedades.

Evaluación de la producción de la leche diaria. Se registró en planillas, pesándose en balanza de $10 \mathrm{~kg}$, después de cada ordeño (mañana y tarde), el promedio de producción de leche diaria por $\mathrm{VMH}$, se determinó entre la cantidad total de leche producida, con el número de días de cada tratamiento. Las tres raciones fueron analizadas bromatológicamente, al inicio, proceso y al final. ${ }^{16}$

La cantidad de agua requerida para producir $1 \mathrm{~kg}$ de leche. El consumo de agua de las $\mathrm{VMH}$, todos los días durante la investigación fue al libitum, con excepción de los días fijados para el cálculo del consumo de agua de las $9 \mathrm{VMH}$, tres veces durante toda la investigación, inicio, proceso y al final. ${ }^{17}$

\section{Resultados}

Tabla 2 Consumo promedio de pradera nativa (MS) por las vacas mestizo Holstein

\begin{tabular}{cccc}
\hline Nr Muestras & $\begin{array}{c}\text { Consumo promedio de } \\
\text { MS por } \mathbf{~ m}^{\mathbf{2}}(\mathbf{k g})\end{array}$ & $\begin{array}{c}\text { Área total pastoreada } \\
\left(\mathbf{m}^{2}\right)\end{array}$ & Consumo de MS (kg/día) \\
\hline $\mathbf{3}$ & 0.2 & 36.3 & 7.1 \\
\hline
\end{tabular}

Tabla 3 Composición química de los insumos alimenticios y pradera nativa

\begin{tabular}{lcccc}
\hline Nutrientes & Unidad & Borra de cerveza & Maíz amarillo partido & Pradera nativa \\
\hline Materia seca & $\%$ & 28.3 & 88.2 & 30.3 \\
Proteína cruda & $\mathrm{g} / \mathrm{kg}$ & 121.2 & 75.2 & 79.1 \\
Energía metabolizable & $\mathrm{Mcal} / \mathrm{kg}$ & 0.7 & 2.4 & 1.2 \\
Calcio & $\mathrm{g} / \mathrm{kg}$ & 0.3 & 0.6 & 1.6 \\
Fosforo & $\mathrm{g} / \mathrm{kg}$ & 0.8 & 2.8 & 6.7 \\
\hline
\end{tabular}

Tabla 4 Ración formulada para cada tratamiento en vacas mestizo Holstein

\begin{tabular}{ccccc}
\hline Tratamientos & Alimentos & \% MS & TCO kg & MS kg \\
\hline Ración I (Sin suplemento) & Pradera nativa & 30.30 & 23.60 & 7.15 \\
& Pradera nativa & 30.30 & 23.60 & 7.15 \\
Ración II (testigo) & Borra de cerveza & 28.29 & 6.00 & 1.70 \\
& Pradera nativa & 30.30 & 23.60 & 7.15 \\
\multirow{2}{*}{ Ración III (balanceado) } & Borra de cerveza & 28.29 & 1.58 & 0.45 \\
& Maíz amarillo & 88.22 & 5.48 & 4.81 \\
\hline
\end{tabular}


Tabla 5 Relacion de las raciones formuladas a las vacas mestizo Holstein

\begin{tabular}{|c|c|c|c|c|c|c|}
\hline Ración formulada & MS (kg) & Pc (g) & EM (Mcal) & $\mathrm{Ca}(\mathrm{g})$ & $\mathbf{P}(\mathrm{g})$ & TCO $(\mathrm{kg})$ \\
\hline \multicolumn{7}{|l|}{ Ración I } \\
\hline Pradera nativa & 7.15 & 565.63 & 8.94 & 11.44 & 47.77 & 23.60 \\
\hline Total en alimento & 7.15 & 565.63 & 8.94 & 11.44 & 47.77 & 23.60 \\
\hline Total requerimiento & 11.25 & 982.00 & 20.60 & 38.76 & 25.24 & \\
\hline Déficit/exceso & -4.10 & -416.37 & -11.66 & -27.32 & +22.53 & \\
\hline \multicolumn{7}{|l|}{ Ración II } \\
\hline Pradera nativa & 7.15 & 565.63 & 8.94 & 11.44 & 47.77 & 23.60 \\
\hline Borra de cerveza & 1.70 & 205.72 & 1.14 & 0.44 & 1.39 & 6.00 \\
\hline Total en alimento & 8.85 & 771.35 & 10.08 & 11.88 & 49.16 & 29.60 \\
\hline Total requerimiento & 11.25 & 982.00 & 20.60 & 38.76 & 25.24 & \\
\hline Déficit/exceso & -2.40 & -210.65 & -10.52 & -26.88 & +23.92 & \\
\hline \multicolumn{7}{|l|}{ Ración III } \\
\hline Pradera nativa & 7.15 & 565.63 & 8.94 & 11.44 & 47.77 & 23.60 \\
\hline Borra de cerveza & 0.45 & 54.36 & 0.30 & 0.00 & 0.00 & 1.59 \\
\hline Maíz amarillo partido & 4.81 & 362.01 & 11.36 & 0.00 & 0.00 & 5.46 \\
\hline Total en alimento & 12.41 & 982.00 & 20.60 & 11.44 & 47.77 & 30.64 \\
\hline Total requerimiento & 11.25 & 982.00 & 20.60 & 38.76 & 25.24 & \\
\hline Déficit/exceso & 1.16 & 0.00 & 0.00 & -27.32 & 22.53 & \\
\hline
\end{tabular}

Tabla 6 Análisis de varianza para la producción de la leche para las vacas mestizo Holstein suplementadas con borra de cerveza y maíz amarillo

\begin{tabular}{lccccc}
\hline Fuente de Variación & G.L. & S.C. & C.M. & Valor F & Pr > F \\
\hline Raciones & 2 & 1598.18 & 799.09 & 213.78 & $<.01 * *$ \\
Error & 717 & 2680.09 & 3.74 & & \\
Total & 719 & 4278.26 & & & \\
\hline CV $22.05 \%$ & & & &
\end{tabular}

Figura 1 Comparación de medias Duncan para la producción de la leche de las vacas mestizo Holstein alimentadas con tres raciones

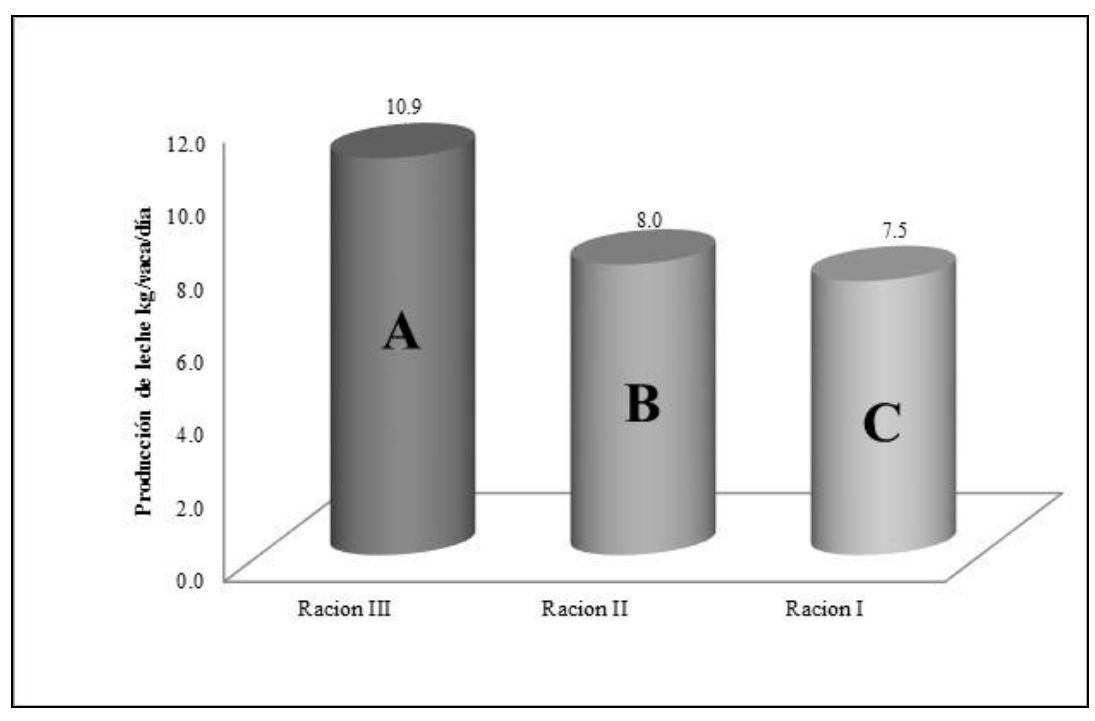


Tabla 7 Contenido de proteína, grasa y solidos totales en la leche de las vacas mestizo Holstein alimentadas con tres diferentes raciones $(\%)$

\begin{tabular}{lcccccc}
\hline Tratamientos & Descripción & Nr & Promedio & DS $^{ \pm}$ & Max & Min \\
\hline Ración I & \multirow{2}{*}{ Proteína } & 3 & 3.60 & 1.69 & 5.19 & 1.83 \\
Ración II & 3 & 4.32 & 1.80 & 6.23 & 2.66 \\
Ración III & & 3 & 4.06 & 0.93 & 4.76 & 3.00 \\
\hline Ración I & & 3 & 2.91 & 0.27 & 3.10 & 2.60 \\
Ración II & \multirow{2}{*}{ Grasa } & 3 & 3.50 & 0.79 & 4.40 & 2.90 \\
Ración III & & 3 & 3.18 & 0.69 & 3.96 & 2.67 \\
\hline Ración I & & 3 & 12.69 & 0.63 & 13.16 & 11.98 \\
Ración II & \multirow{2}{*}{ Sólidos totales } & 3 & 13.50 & 0.73 & 14.27 & 12.81 \\
Ración III & & 3 & 11.34 & 0.49 & 11.87 & 10.90 \\
\hline
\end{tabular}

Tabla 8 Análisis de varianza para el contenido de proteína, grasa y solidos totales en la leche cruda de las vacas mestizo Holstein suplementadas con borra de cerveza y maíz amarillo

\begin{tabular}{|c|c|c|c|c|c|}
\hline Fuente de Variación & G.L. & S.C. & C.M. & Valor F & $\operatorname{Pr}>\mathbf{F}$ \\
\hline \multicolumn{6}{|l|}{ Proteína } \\
\hline Ración & 2 & 4.86 & 2.43 & 0.91 & $0.45 \mathrm{NS}$ \\
\hline Error & 6 & 16.11 & 2.68 & & \\
\hline Total & 8 & 20.98 & & & \\
\hline \multicolumn{6}{|l|}{ Grasa } \\
\hline Ración & 2 & 0.53 & 0.26 & 0.69 & $0.54 \mathrm{NS}$ \\
\hline Error & 6 & 2.34 & 0.39 & & \\
\hline Total & 8 & 2.88 & & & \\
\hline \multicolumn{6}{|l|}{ Solidos totales } \\
\hline Ración & 2 & 7.18 & 3.59 & 9.22 & $0.01 *$ \\
\hline Error & 6 & 2.33 & 0.38 & & \\
\hline Total & 8 & 9.52 & & & \\
\hline
\end{tabular}

Figura 2 Comparación de media DUNCAN para el contenido de sólidos totales en la leche de las vacas mestizo Holstein suplementadas con borra de cerveza y maíz amarillo (\%)

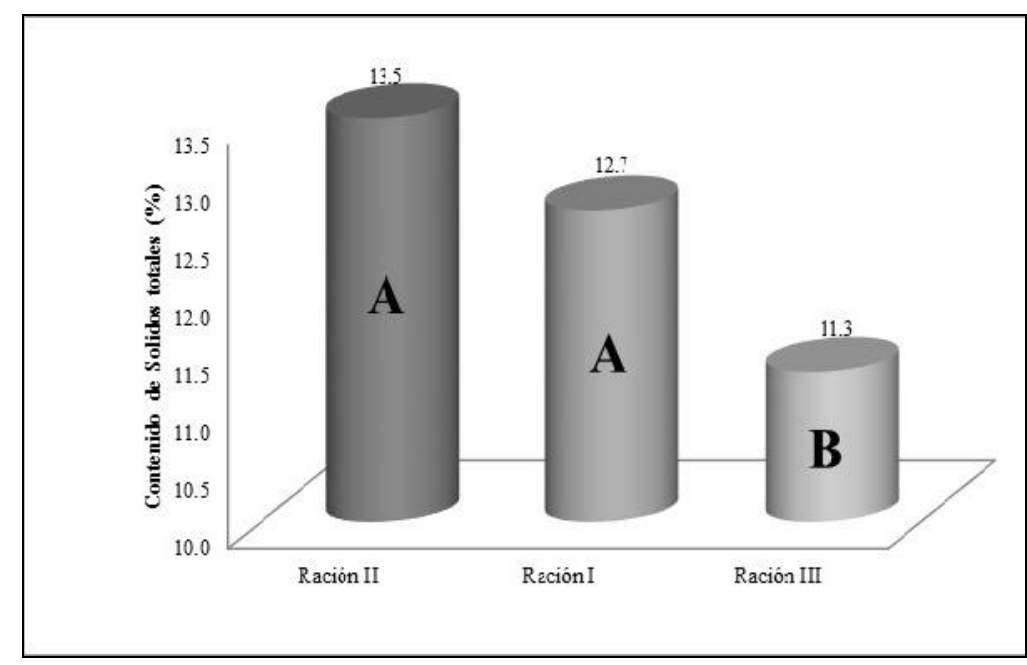


Tabla 9 Cantidad de agua requerida para producción un kg de leche en las vacas mestizo Holstein suplementadas con borra de cerveza y maíz amarillo

\begin{tabular}{lcc}
\hline \multicolumn{1}{c}{ Tratamiento } & $\mathbf{N}^{\circ}$ de repeticiones & Cantidad de agua requerida para $(\mathbf{L})$ \\
\hline Ración II & 3 & 16.2 \\
Ración I & 3 & 13.0 \\
Ración III & 3 & 12.0 \\
\hline
\end{tabular}

Tabla 10 Costos totales de producción de la leche suplementados con borra de cerveza y maíz amarillo

\begin{tabular}{|c|c|c|c|c|}
\hline \multirow[b]{2}{*}{ Tratamientos } & \multirow[b]{2}{*}{ Costos fijos (Bs) } & \multicolumn{2}{|c|}{ Costos variables $(\mathrm{Bs})$} & \multirow{2}{*}{$\begin{array}{c}\text { Costos } \\
\text { totales (Bs) }\end{array}$} \\
\hline & & $\begin{array}{c}\text { Durante la } \\
\text { crianza }\end{array}$ & Costo alimento & \\
\hline Ración I & 500.16 & 1851.46 & 719.97 & 3071.59 \\
\hline Ración II & 500.16 & 1851.46 & 950.37 & 3301.99 \\
\hline Ración III & 500.16 & 1851.46 & 3146.37 & 5497.99 \\
\hline
\end{tabular}

Tabla 11 Costos de producción de la leche en las vacas mestizo Holstein alimentadas con tres raciones

\begin{tabular}{cccc}
\hline Tratamientos & $\begin{array}{c}\text { Costos totales } \\
(\mathbf{B s})\end{array}$ & $\begin{array}{c}\text { Producción de } \\
\text { leche }(\text { total/kg) }\end{array}$ & $\begin{array}{c}\text { Costo unitario } \\
(\mathbf{B s} / \mathbf{K g})\end{array}$ \\
\hline Ración I & 3071.59 & 1803.3 & $\mathbf{1 . 7 0}$ \\
Ración II & 3301.99 & 1908.0 & $\mathbf{1 . 7 3}$ \\
Ración III & 5497.99 & 2606.3 & $\mathbf{2 . 1 0}$ \\
\hline
\end{tabular}

\section{Discusión}

La pradera nativa (PN) del municipio de Pucarani presentó una oferta de forraje para el pastoreo, cuyas especies nativas son utilizadas por las UFP's, de forma cotidiana, con el fin de proveer alimentación a su ganado, fue de $7.1 \mathrm{~kg} / \mathrm{MS} /$ día (tabla 2), la MS de la PN fue $30.3 \%$ (tabla 3), y el consumo en materia verde (TCO) fue de $23.6 \mathrm{~kg} /$ día/vaca (tabla 4). Según Rivera-Calderón ${ }^{18}$, señala que 5.48 $\mathrm{kg} / \mathrm{MS} /$ día, que difieren de nuestros resultados, aquí debemos considerar que estas diferencia pueden asociarse a factores que influyen en la palatabilidad, como preferencia del animal, su preñez, lo que se relaciona con la estación del año, estado de crecimiento del forraje, clima, y las características de la planta. ${ }^{19}$
En tanto, Huss el al. ${ }^{20}$, señala, un síntoma importante en la deficiencia de proteína, es la falta de apetito, dando lugar a la disminución de energía, siendo la suplementación proteica un estímulo al consumo de forraje con alto contenido de lignocelulosa, aumentando el consumo de energía, que explicaría, que los animales consuman en épocas secas plantas lignificadas.

La tabla5, refiere al balance energético, la ración III, MA y BC, cubren los requerimientos energéticos en cuanto a PC, EM y MS, en tanto el PPN y la ración II, no cubren los requerimientos que otorga la ración III. De ahí que se hace necesario, orientar a los productores del cordón lechero y quizá otras zonas que se dedican a esta actividad, a realizar estudios pre- 
liminares, cuyo fin sea evaluar estos parámetros de benéfico, en sus actividades pecuarias.

Cabe señalar que trabajos de esta naturaleza en la zona de estudio son de escaso acceso o bien, no están disponibles para los autores de la presente investigación.

El análisis de varianza (ANVA) tabla 6, las diferencias altamente significativas $(\mathrm{P}<0.05)$ para el factor raciones, con un coeficiente de variación de 22.05 $\%$, la figura 1, comparación de medias Duncan, como se había señalado anteriormente, cubrir la energía necesaria para los procesos metabólicos depende de la dieta, la producción de leche su cantidad y calidad se relacionan con el aporte proteico y energía en la dieta en lactación. ${ }^{21}$ La energía necesaria para el metabolismo de rumiantes proviene básicamente de ácidos grasos volátiles (AGV), producidos en el rumen, ahora, la composición de la dieta a nivel ruminal ocurrirá una variación entre las proporciones de ácido acético/butírico, precursores de grasa y ácido propiónico, precursor la lactosa, responsable del volumen de leche producida.

En el rumen, la dieta puede cambiar la población microbiana, como consecuencia influir la producción de $\mathrm{AGV}$, dietas altas en fibra producen $\mathrm{AGV}$ con elevada proporción de ácido acético, en tanto, dietas de concentrado producen más ácido propiónico, cambios que afectarían la composición de la leche, así, la proporción molar de ácido acético/butírico están relacionados directamente con la concentración de grasa en la leche, empero, un incremento en la proporción de ácido propiónico ha producido una mejor partición de la energía, la que refleja en su tejido corporal y menos en la leche. ${ }^{22}$ El incremento en la producción de leche por la suplementación con BC y MA, puede atribuirse a que durante la alimentación, con la ración I, las VMH fueron alimentadas exclusivamente en el sistema de 73 pastoreo tradicional en estaca, sin ningún suplemento, consumiendo una cantidad de $7.15 \mathrm{~kg}$ de MS, que aportan $565.63 \mathrm{~g}$ de PC y 8.94 Mcal de EM, que no satisfice sus requerimientos, así una VMH produce en promedio $8.1 \mathrm{~kg}$ de leche con un requerimiento de $982.00 \mathrm{~g}$ de PC, $20.60 \mathrm{Mcal}$ de EM y $11.25 \mathrm{~kg} / \mathrm{MS} /$ día.

Mientras las vacas alimentadas con la ración II (testigo) pastoreadas en la misma superficie de PN, fueron suplementados con $1.70 \mathrm{~kg} / \mathrm{MS}$ de BC, les aportó 205.72 g de PC y 1.14 Mcal de EM, que les aportó $771.35 \mathrm{~g}$ de proteína y $10.08 \mathrm{Mcal}$ de energía, mejorando el aporte de proteína, como energía, sin embargo aun así, no satisficieron sus requerimientos, pero incrementó la producción de leche, esto se debe a la mejora proteica en su alimentación. Con la ración III (balanceado), las VMH, suplementados con $0.45 \mathrm{~kg}$ de MS de BC aportó $54.36 \mathrm{~g}$ de proteína, 0.30 Mcal de energía y $4.81 \mathrm{~kg}$ de MS de MA que aportó $362.01 \mathrm{~g}$ de proteína y $11.36 \mathrm{Mcal}$ de energía, llegando a producir $8.1 \mathrm{~kg}$ de leche, y necesitan $982.00 \mathrm{~g}$ de proteína, 20.60 Mcal de energía y una cantidad de $11.25 \mathrm{~kg} / \mathrm{MS}$. Por tanto, con respecto al incremento de la producción de leche con la ración III, se atribuye a que existió un equilibrio de nutrientes de proteína y energía cubriendo así sus requerimientos necesarios, siendo que la producción de leche incrementó en $31.20 \%$ en comparación a la ración I.

Patty-Quispe et al. ${ }^{15}$, Señalan, la producción de leche en vacas PPN el suplemento concentrado a base de MA partido, afrechillo de trigo, sal común y sal mineral, logran obtener en el periodo de testigo $3.42 \mathrm{~kg} /$ día, adaptación $5.11 \mathrm{~kg}$ /día y con suplementación $6.24 \mathrm{~kg} / \mathrm{día}$, en comparación las vacas suplementadas con heno fortificado en el periodo de testigo con $3.58 \mathrm{~kg} /$ día, adaptación $3.94 \mathrm{~kg} /$ día y suplementación 4.69 kg/día. 
Tonconi $^{23}$, señala que el heno de alfalfa mejora la producción promedio de leche con $7.60 \mathrm{~kg} / \mathrm{vaca} /$ día, germen de maíz $6.10 \mathrm{~kg} / \mathrm{vaca} /$ día, jipi-broza de quinua $5.6 \mathrm{~kg} / \mathrm{vaca} /$ día y $\mathrm{PN}$ en descanso 5.20 $\mathrm{kg} / \mathrm{vaca} /$ día, este último inferior a los anteriores.

Nuestros datos si bien no son coincidentes con estos autores, puede deberse a que la suplementación, otorgada buscaba, el complementar los requerimientos nutricionales, que en la tabla 5, señalan que en la ración I y II, no lograron abastecer sus requerimientos, así la ración III logro evitar ese déficit, lo que nos da pauta a que en futuros trabajos de investigación, se hace necesario establecer como condicionante, una evaluación previa y a partir de esta, realizar esquemas nutricionales, que en la medida que se pueda sean aporte a las mejoras en la producción de leche, pero que a su vez la calidad de esta no sea alterada, ya que afectaría en su comercialización hacia los centros de abasto.

Si nos referimos a la calidad de la leche, las evaluaciones de contenido de proteína, grasa y solidos totales ${ }^{24}$, tabla 7 y 8 , indica que las diferencias en el contenido de proteína en la leche explicaría que la adición del tipo de ración, afectara al porcentaje de proteina, ya que se forma a partir de los aminoácidos de la proteína microbiana y de la dieta que son digeridas y absorbidos a nivel del intestino delgado. Wattiaux ${ }^{25}$, menciona que la concentración de proteína varía de 3.0 a $4.0 \%$ (30-40 g/L), esto varía con la raza, existiendo una estrecha relación entre la cantidad de grasa y proteína en la leche, cuanto mayor sea la cantidad de grasa, mayor es la cantidad de proteína.

Agudelo-Gómez \& Bedoya-Mejía ${ }^{26}$, indica que la proteína contenida en la leche es de 3.5\% (variando desde el 2.9\% al 3.9\%). "Esta proteína láctea" una mezcla de numerosas fracciones proteicas diferentes, de pesos moleculares distintos. En la alimentación con ración I, las VMH el porcentaje de proteína se ve afectada por mayor cantidad de déficit de proteína en la ración, a causa del contenido ruminal, que es pésimo para generar aminoácidos, los cuales juegan un rol muy importante en el \% de proteína de la leche.

Mientras la alimentación con la ración II, las VMH, ya que se suplemento con fuente de proteína cruda, mejoró la cantidad de proteína en su alimentación, y consecuentemente, mejoro el contenido ruminal para producir mayor cantidad de aminoácidos, como resultado se tendría la mejora del \% de proteína en la leche. Las VMH con la ración III, se logró cubrir su requerimiento de proteína cruda con 982 g, lo que equilibro la proteína en su dieta para mejorar el contenido de proteína ruminal, y a partir de este nutriente se forme mayor cantidad de aminoácidos, y así puedan formar e incrementar la proteína de la leche cruda.

$\mathrm{Al}$ respecto Hernandez \& Ponce $^{27}$, mencionan, cuando existe déficit de proteína en la alimentación, se ve afectada el contenido del porcentaje de grasa y los sólidos totales. Wattiaux ${ }^{25}$, indica que normalmente la grasa constituye desde el 3.5 al $6.0 \%$ de la leche, variando entre la raza de los animales y las prácticas de alimentación. Una ración demasiado rica en concentrados que no estimulan la rumia, puede resultar en una caída 2.0 a $2.5 \%$ de grasa. Lactodata $^{28}$, afirma que los rumiantes requieren cierta cantidad de fibra para estimular la función del rumen y mantener el nivel de grasa láctea, de la misma manera nos indica que el valor óptimo de fibra cruda de la materia seca deberá estar entre el 17 y $22 \%$, si los valores superan el $22 \%$ de fibra cruda, la capacidad de consumo de alimento podría perjudicarse, si se ofrece por debajo del $17 \%$ de fibra cruda el nivel de grasa de la leche se reduce. Andrade ${ }^{29}$, indica que la riqueza de la leche en grasa varía con el periodo de lactancia, la época del año, 
el tiempo entre ordeñas, la edad y sobre todo según los alimentos que integran la ración.

PDLA $^{30}$, menciona que el porcentaje de grasa aceptado por las industrias lácteas como mínimo es del $2.8 \%$, a mayor contenido de grasa (mayor a $4 \%$ ), se tiene en la leche mayor rendimiento de queso, es decir de $5 \mathrm{~L}$ de leche con $4 \%$ de grasa se obtiene 1 $\mathrm{kg}$ de queso. La alimentación con la ración I, existe déficit de proteína cruda, lo que afecta el contenido de porcentaje de grasa de la leche. Mientras la alimentación con la ración II, hay aumento gradual en el contenido de la grasa, lo que se atribuye por el aumento en la producción y absorción del ácido acético a través del rumen. Las VMH se ve la reducción mínima del porcentaje del contenido de la grasa en comparación a la ración II, esto quizás se deba a que deficiencia de fibra en su alimentación.

IBNORCA $^{31}$, indica, el porcentaje de sólidos totales mínimo aceptado por la Norma Boliviana es $10.8 \%$, mismo que es el resultado de grasa, lactosa, caseína, albumina de las sales minerales, lo nos permite reconocer si la leche es de buena calidad. Por otro lado Chura $^{32}$, indica, el contenido de sólidos totales, es superior en la época húmeda con $12 \%$, e inferior en la época seca con $11.8 \%$. Estas diferencias en el contenido de sólidos totales, se pueden atribuir, a la genética, raza, alimentación y etapa de lactancia. Durante la época húmeda existen forrajes de buena calidad nutricional (alfalfa, avena, cebada y pastos naturales) que influyen para que el contenido de sólidos totales, mientras su disminución, durante la época seca, están determinadas por la poca disponibilidad de forrajes.

$\mathrm{Al}$ respecto Saborío-Montero ${ }^{33}$, señala que la leche está constituida en un 85-90\% por agua, el 10-15\% restante es lo que se conoce como ST y está conformada principalmente por lactosa, grasa, proteína y minerales, entre los factores más significativos 75 que afectan en el contenido de sólidos de la leche son: la raza, dieta, época del año, disponibilidad y calidad del alimento, producción de leche y etapa de lactancia, contenido de células somáticas. Razón que puede explicar que el resultado obtenido de sólidos totales en la presente investigación, atribuyéndose a las raciones, que sería afectado por factores como, genética, volumen de producción y otros. La variación que existe entre los promedios del \% ST de la leche en VMH con la ración I, se debe al no ser suplementado con ningún tipo de insumo alimenticio por lo que, la PN no cubre los requerimientos de PC, afectando directamente el \% de ST. Mientras las VMH suplementadas con la $\mathrm{BC}$ el incremento del \% ST de la leche, se atribuiría por el aumento de PC en la ración, lo que juega un rol muy importante en la variación del porcentaje de ST. Finalmente las VMH con la ración III, se reduce el contenido de ST de leche, esta diferencia de atribuye al balanceo de los nutrientes (PC y EM) en la alimentación de las vacas y por consiguiente menor producción de ácido propiónico afectando en la síntesis de lactosa y proteína láctea, sumado a esto el incremento en el volumen de producción de la leche.

\section{Conflictos de intereses}

En el manejo de los animales se ha cumplido normas éticas. Esta investigación ha sido autofinanciada por los autores y no genera conflictos de interés.

\section{Agradecimientos}

A la directiva de la Asociación de Lecheros Vacunos de Patamanta (ALVAPA), por proporcionarnos las vacas mestizo Holstein. Al Instituto de Servicios de Laboratorios de Diagnostico e Investigación en 
Salud (SELADIS) por los análisis de las muestras evaluadas en la presente investigación.

\section{Literatura citada}

1. Organización de las Naciones Unidas para la Alimentación y la Agricultura (FAO). Federación Panamericana de Lechería (FEPALE). Situación de la lechería en America Latina y el Caribe 2011. Informe producido en el ámbito del observatorio de la cadena láctea de América Latina y el Caribe; 2011.

2. Cortez Quispe H, Paredes Alvarado D, Cabrera Gutiérrez C, Alarcón Catarí E. Producción lechera y efectos del cambio climático en dos comunidades del Altiplano Norte. Centro de Investigación y Promoción del Campesinado (CIPCA). La Paz, Bolivia; 2014.

3. Quimo Relova EG. Determinación de los costos de producción de leche y derivados lácteos a nivel artesanal en las Provincias los Andes, Murillo y Omasuyos del Departamento de La Paz [Tesis Licenciatura]. La Paz: Universidad Mayor de San Andrés; 2008. p. 106.

4. Centro de Estudios para el Desarrollo Laboral y Agrario (CEDLA. Producción campesina y mercados. La ganadería lechera en el Altiplano de La Paz. CEDLA, PROCADE UNITAS. La Paz, Bolivia; 1997. p. 19-76.

5. Vélez Terranova OM. Análisis de las limitaciones nutricionales y de manejo en un sistema de producción lechera en el Valle del Cauca [Tesis Maestría]. Cauca: Universidad Nacional de Colombia; 2011.

6. Gaviria Uribe X, Naranjo Ramírez JF, Bolívar Vergara DM, Barahona Rosales R. Consumo y digestibilidad en novillos cebuínos en un sistema silvopastoril. Arch Zootec 2015;64(245):21-7. http://dx.doi.org/10.21071/az.v64i245.370

7. National Research Council (NRC). Nutrients requirements of dairy cattle. 7th ed. National Academy Press, WA, USA; 2001.

8. Sánchez Chopa F, Nadin LB, Gonda HL. Two drying methods of bovine faeces for estimating $n$ alkane concentration, intake and digestibility: A comparison. Anim Fee Sci Technol 2012;177(12):1-6. https://doi.org/10.1016/j.anifeedsci.2012. $\underline{06.003}$

9. Kerr DV, Cowan RT, Chaseling J. Estimations of the increase in milk production due to the introduction of maize silage to a dairy farm in a subtropical environment: A time series approach. Agric Syst 1991;35(3):313-20. https://doi.org/10. 1016/0308-521X(91)90161-3

10. Cowan R. Dairy feeding systems based on pasture and forage crop in the tropic and subtropic. Armidale, NSW Workshop, CSIRO; 2005. p. 3-6.

11.Davis LD. Sistemas de alimentación para optimizar la rentabilidad de rebaño lecheros de alta productividad en EEUU; 2008. (en línea). Consultado 12 agosto 2017. http://www.produc cionAnimal.com.ar/producción_bovina_de_leche /producción_bovina_leche/81-alimentacion.pdf.

12.Fundación Española para el Desarrollo de la Nutrición Animal. (FENDA). Tablas FENDA de composición y valor nutritivo de forrajes y subproductos fibrosos húmedos. España. 2010. Disponible en: http://www.fundacionfedna.org/ ingredientes-para-piensos

13. Alcázar J. Bases para la alimentación animal y la formulación manual de raciones. La Paz, Bolivia. Ed. Génesis; 1997. p. 35-71.

14.Plan de Desarrollo Municipal del Gobierno Autónomo Municipal de Pucarani (PDMP); (20072010). 
15.Patty-Quispe MH, Loza-Murguia MG, AchuNina C, Rojas-Pardo A, Chura-Limachi F, Quispe-Paxipati $\mathrm{CH}$. Evaluación del efecto de suplemento de heno fortificado y concentrado en la producción de leche de bovinos (Bos taurus L.) durante la época seca en la comunidad AchacaTiahuanacu. J Selva Andina Anim Sci 2017;4(1):13-37.

16. American Organization of Analytical Chemists International (AOAC). Official methods of analysis. 15th ed. Arlington, VA, USA: Association of Official Analytical Chemists; 1990.

17. Ríos Ramirez JN. Comportamiento hidrológico de sistemas de producción ganadera convencional y silvopastoril en la zona de recarga hídrica de la subcuenca del Rio Jabonal, cuenca del Rio Barranca, Costa Rica [Tesis Maestría]. Turrialba: CR CATIE; 2006. p. 116.

18. RiveraCalderón H. Evaluación de la capacidad de carga animal en unidades familiares de producción de la comunidad de Avichaca, en dos épocas del año [Tesis Licenciatura]. La Paz: Universidad Mayor de San Andrés; 2007. p. 108.

19.Flores A, Malpartida E. Manual de forrajes para zona árida y semiáridas andinas. Universidad de california instituto nacional de investigación Lima Perú; 1992. p. 20-25.

20.Huss D, Bernardón A, Anderson D, Brun J. Principios de manejo de pasturas. FAO; 1996. p. 83110.

21.Pereira MM, Armentano LE. Partial replacement of forage with nonforage fiber sources in lactating cow diets. II. Digestion and rumen function. J Dairy Sci 2000;83(12):2876-87.

22. Agnew RE, Yan T. Impact of recent research on energy feeding systems for dairy cattle. Livest Prod Sci 2000;66(3):197-215.
23.Tonconi S. Evaluación de costos y producción de leche en vacas mestizas por efecto de tres suplementos alimenticios en la Unidad Académica Campesina-Tiahuanacu departamento de La Paz [Tesis Licenciatura]. La Paz: Universidad Católica Boliviana San Pablo; 2015.

24.Bach A, Calsamiglia S. La fibra en los rumiantes: ¿Quimica o Fisica?. 2006. Recuperado el 11 de junio de 2015, de la fibra en los Rumiantes http://www.produccionovina.com.ar/informacion tecnica/manejo del alimento/100.

25.Wattiaux M. Alimentos para vacas lechera. 2012. (en línea). Consultado 16 marzo 2015 Disponible.en:http://www.babcock.wiaw.edu/pub lication/overview/de.es.lazo.com.

26.Agudelo-Gómez DA, Bedoya-Mejía O. Composición nutricional de la leche de ganado vacuno. Rev Lasallista Investig 2005;2(1):38-42.

27.Hernández R, Ponce P. Efecto de tres tipos de dietas sobre la aparición de trastornos metabólicos y su relación con alteraciones en la composición de la leche en vacas Holstein Friesian. Zoot Tropical 2005;23(3):295-310.

28.Lactodata. Comisión nacional del sistema producto bovinos leche. 2015. (en línea) disponible www.lechemexico.org.mx/lactodata/leche/index. php (obtenido el 5 de agosto del 2015).

29. Andrade L. Influencia de la paja de cebada (Hordeum vulgare) y Chillihua (Festuca dolichophylla) tratadas con urea en la producción de leche y peso vivo en vacas mestizas del altiplano central [Tesis Licenciatura]. La Paz: Universidad Mayor de San Andrés; 2002. p. 92.

30.Programa de Desarrollo Lechero del Altiplano (PDLA). Alimentación y nutrición del ganado lechero. Manual de auto instrucción. Ministerio de Agricultura, Ganadería y Desarrollo Rural; 2005. 
31.Instituto Boliviano de Normalización y Calidad Alimenticia (IBNORCA). EQNB-33013. Norma Boliviana. Requisitos físico-Químicos; 2004. p. 8.

32. Chura F. Evaluación de la calidad de leche bovina en época seca y húmeda, en el municipio de Tiahuanacu, provincia Ingavi- Departamento de La Paz [Tesis Licenciatura].La Paz: Universidad Católica Boliviana San Pablo; 2006.
33. Saborío-Montero A. Factores que influencian el porcentaje de sólidos totales de la leche Zootecnista. Centro de Investigaciones en Nutrición Animal. Universidad de Costa Rica; 2011. 